\title{
CTCF: from insulators to alternative splicing regulation
}

\author{
Alberto R Kornblihtt ${ }^{1}$ \\ ${ }^{1}$ Laboratorio de Fisiología y Biología Molecular, Departamento de Fisiología, Biología Molecular y Celular, IFIBYNE-CONICET, \\ Facultad de Ciencias Exactas y Naturales, Universidad de Buenos Aires. Ciudad Universitaria, Pabellón 2, (C1428EHA) Buenos \\ Aires, Argentina \\ Cell Research (2012) 22:450-452. doi:10.1038/cr.2012.22; published online 7 February 2012
}

The zinc-finger DNA-binding protein CTCF has been known for being a constituent of insulators. A recent paper in Nature reports an unforeseen intragenic role for CTCF that links DNA methylation with alternative splicing. By binding to its target DNA site placed within an alternative exon, CTCF creates a roadblock to transcriptional elongation that favors inclusion of the exon into mature mRNA. DNA methylation prevents CTCF binding, which releases pol II transient blockage and promotes exon exclusion.

One of the most exciting aspects of scientific research is not discovery per se but the fall of preconceptions. In the history of the knowledge of the flow of gene expression in eukaryotic cells, many preconceptions have fallen. Perhaps the most striking one is that eukaryotic genes are not contiguous with their corresponding mRNAs, as thought in the 60's and early 70's of the last century, but split in exons and introns, which calls for the splicing mechanism that gets rid of the introns from a precursor mRNA and joins the exons to give rise to the mature, translatable mRNA molecule. Soon after the discovery of splicing in 1976, alternative splicing was reported, but only recently we

Correspondence: Alberto R Kornblihtt

E-mail: ark@fbmc.fcen.uba.ar learnt that this mechanism, responsible for the generation of a vast proteomic diversity from a limited number of genes, seems to be present in $90 \%$ of the approximately 23000 human genes, which also represents an unexpected surprise compared to previous estimations of $20 \%$. A third preconception that has been violated more recently is that splicing is a post-transcriptional event, and therefore independent from transcription. On the contrary, not only splicing is mostly co-transcriptional but both splicing and alternative splicing are coupled to transcription and in consequence, splicing is not only affected by the splicing machinery but also by the chromatin structure and factors regulating the transcription process, such as promoters, transcription factors and co-activators, which may affect either the speed of RNA polymerase II (pol II) elongation or the recruitment of splicing factors to the transcription apparatus (for reviews see [1, 2]). Differential transcriptional elongation rates inside genes create different windows of time for the splicing machinery to be recruited to and act on relevant sequences as they are present in the nascent pre-mRNA [3].

A role for chromatin in the control of intragenic elongation rates and in consequence, in alternative splicing has been demonstrated by several groups who used drugs or signal cascades that promote either more compact or more relaxed chromatin configurations that impede or facilitate elongation, respectively. For example, trichostatin A $[4,5]$, recruitment to promoters of histone acetyltransferases [6] and neuron depolarization [7] regulate alternative splicing because of chromatin relaxation promoted by histone acetylation. Consistently, the transcription-associated $\mathrm{H} 3 \mathrm{~K} 36 \mathrm{me} 3$ modification is less prominently enriched in alternatively spliced exons than in constitutive exons [8]. In an opposite way, chromatin remodelers such as SWI/SNF [9] or siRNAs that trigger silencing histone marks such as H3K9me2 and H3K27me3 through a argonaute-1-mediated transcriptional gene silencing mechanism [10] were shown to create local chromatin compactions that act as roadblocks to pol II elongation, in turn affecting alternative splicing decisions. Furthermore, several genome-wide studies showed that nucleosomes are preferentially positioned in exons $[11,12,13]$ and this positioning may act as a transient barrier to elongation that helps exon recognition at the RNA level [14, 15]. All together this evidence deeply weakens the preconception that what matters in the study of trancriptional regulation are only promoters and related regulatory elements, by displacing the focus towards the interior of genes where changes in elongation may not affect the total levels of mRNA but deeply change the quality of the message by affecting the propor- 
tions of its splicing isoforms.

It was speculated that DNA methylation may also, indirectly or directly via histone modifications, affect alternative splicing. In fact, DNA methylation patterns correlate well with histone methylation patterns [16] and it was reported that methylation of a DNA sequence in the middle of a gene causes local decreases in histone acetylation and chromatin accessibility, resulting in a decline in pol II elongation, without quantitatively affecting the transcription levels [17]. A recent article in Nature [18] provides compelling evidence for a key role of intragenic DNA methylation in the control of alternative splicing. The authors use the $C D 45$ gene as a model. CD45 is a lymphocyte transmembrane tyrosine phosphatase where inclusion levels of a block of exons 4-6 correlate with lymphocyte development. Heterogeneous ribonucleoprotein hRNPLL is responsible for inhibiting inclusion of exons 4 and 6 but not of exon 5 , whose mechanism of regulation was unknown until Shukla et al. [18] revealed that exon 5 possesses a strong binding site for the zinc-finger DNA-binding protein CTCF (CCCTC-binding factor) and that different lymphocyte cell lines showed a strong positive correlation between exon 5 inclusion and CTCF binding to exon 5. More importantly, RNAi-mediated CTCF depletion leads to reduced exon 5 inclusion. CTCF was conspicuously known for more than 20 years for its sequence-specific binding to insulators, the genetic elements that insulate inactive regions of the genome from active ones and provide boundaries for the action of enhancers. So, what is this "intergenic" protein doing intragenically? Using chromatin immunoprecipitation, Shukla et al. demonstrated that the elongating form of pol II, characterized by phosphorylation of Ser-2 on the YSPTSPS heptad repeats of its carboxy terminal domain (CTD), shows a significant enrichment at CD45 exon 5 DNA but not at exons 4 and 6. Since CTCF depletion not only reduces CTCF binding but also abolishes exon 5 pol II peak, it is concluded that CTCF binding to exon 5 DNA creates a roadblock to pol II elongation. The authors validate their findings both with in vitro transcription of synthetic templates carrying or lacking the CTCF binding site and with transfection of cells in culture with alternative splicing reporter minigenes, demonstrating that CTCF promotes pol II pausing but not complete arrest.

The story turns even more interesting when the link with DNA methylation is investigated. Analysis of several lymphoid cell lines revealed an inverse correlation between CTCF binding and CpG methylation (5-methyl cytosine) at CD45 exon 5. Consistently, inhibition of the maintenance methyltransferase DNMT1 that effectively reduced 5-methyl cytosine levels, also restored CTCF binding, local pol II pausing
A

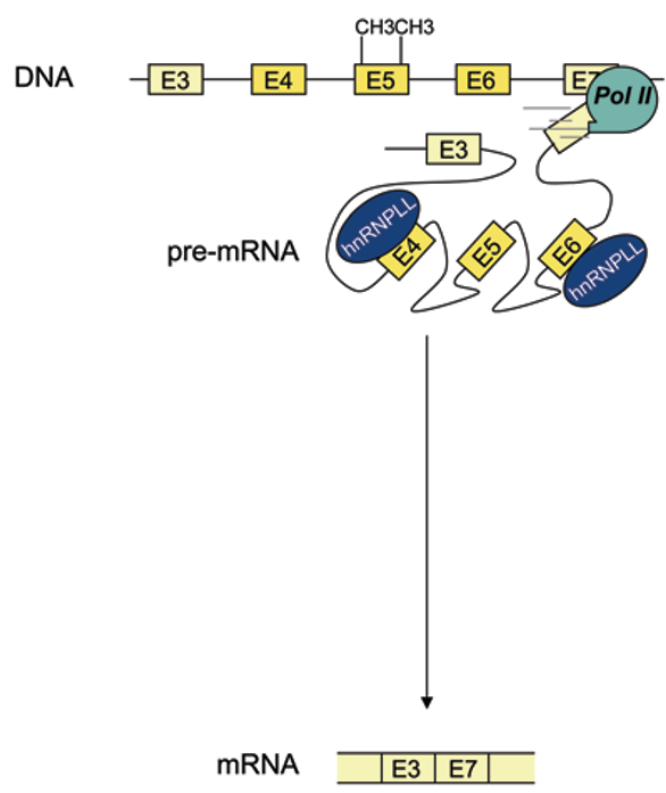

B

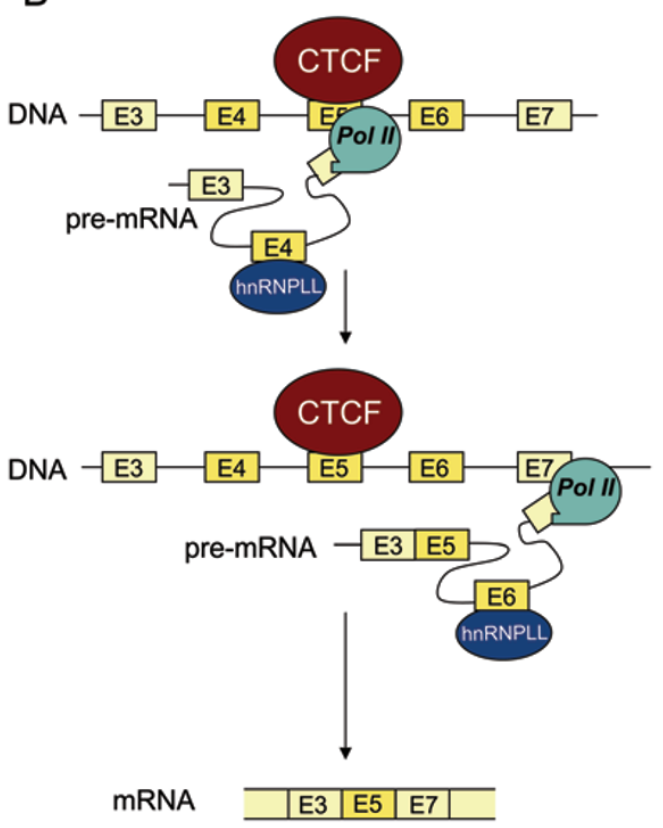

Figure 1 Model for the regulation of $C D 45$ alternative splicing according to Shukla et al. [18]. (A) Methylation of exon 5 DNA prevents binding of CTCF. In the absence of internal pauses, fast elongation promotes exclusion of exon 5 from mature mRNA. Exclusion of exons 4 and 6 is promoted by binding of hnRNPLL to these exons in the pre-mRNA. (B) In the absence of methylation, CTCF binds to exon 5 DNA and creates a transient roadblock to pol II elongation that favors exon 5 recognition at the pre-mRNA level and its inclusion into mature mRNA. Inclusion of exons 4 and 6 remains inhibited by hnRNPL binding. 
and higher exon 5 inclusion. Finally the authors extend their observations with the CD45 gene globally using ChIP-seq and RNA-seq to conclude that intragenic CTCF plays a general role in alternative splicing through its kinetic coupling with transcription.

The paper by Shukla et al. illustrates the roles of DNA methylation and elongation in alternative splicing decisions (Figure 1) that are relevant in the physiology of the immune system. Furthermore, two dogmas are broken by this paper. The first one is the assumption that CTCF only acted between genes and not inside genes. The second one, perhaps more subtle, is that in the mechanism found by Shukla et al., intragenic DNA methylation removes a pause to elongation by preventing the binding of CTCF. As demonstrated by Lorincz et al. [17], intragenic DNA methylation may play the opposite role of blocking elongation. I foresee that these dual opposite roles will greatly depend on the gene context, chromatin structure, and the particular DNA binding proteins that create the pause and the relative proximity or overlapping of the methylation target and the binding site for these proteins. On the other hand, the possibility that certain methylated DNA-biniding proteins regulate splicing via recruitment of splicing factors rather than through the control of elongation as shown recently [19] remains open.

\section{References}

1 Kornblihtt AR. Coupling transcription and alternative splicing. Adv Exp Med Biol 2007; 623:175-189.

2 Perales R, Bentley D. "Cotranscriptionality": the transcription elongation complex as a nexus for nuclear transactions. Mol Cell 2009; 36:178-191.

3 de la Mata M, Alonso CR, Kadener S, et al. A slow RNA polymerase II affects alternative splicing in vivo. Mol Cell 2003; 12:525-532.

4 Kadener S, Cramer P, Nogues G, et al. Antagonistic effects of T-Ag and VP16 reveal a role for RNA pol II elongation on alternative splicing. EMBO J 2001; 20:5759-5768.

5 Nogués G, Kadener S, Cramer P, Bentley D, Kornblihtt AR. Transcriptional activators differ in their abilities to control alternative splicing. $J$ Biol Chem 2002; 277:43110-43114.

6 Auboeuf D, Honig A, Berget SM, O'Malley BW. Coordinate regulation of transcription and splicing by steroid receptor coregulators. Science 2002; 298:416-419.

7 Schor IE, Rascovan N, Pelisch F, Allo M, Kornblihtt AR. Neuronal cell depolarization induces intragenic chromatin modifications affecting NCAM alternative splicing. Proc Natl Acad Sci USA 2009; 106:4325-4330.

8 Kolasinska-Zwierz P, Down T, Latorre I, Liu T, Liu XS, Ahringer J. Differential chromatin marking of introns and expressed exons by H3K36me3. Nat Genet 2009; 41:376-381.

9 Batsché E, Yaniv M, Muchardt C. The human SWI/SNF subunit Brm is a regulator of alternative splicing. Nat Struct Mol Biol 2006; 13:22-29.

10 Alló M, Buggiano V, Fededa JP, et al.
Control of alternative splicing through siRNA-mediated transcriptional gene silencing. Nat Struct Mol Biol 2009; 16:717-724.

11 Schwartz S, Meshorer E, Ast G. Chromatin organization marks exon-intron structure. Nat Struct Mol Biol 2009; 16:990-995.

12 Spies N, Nielsen CB, Padgett RA, Burge CB. Biased chromatin signatures around polyadenylation sites and exons. Mol Cell 2009; 36:245-254.

13 Tilgner $\mathrm{H}$, Nikolaou C, Althammer $\mathrm{S}$, et al. Nucleosome positioning as a determinant of exon recognition. Nat Struct Mol Biol 2009; 16:996-1001.

14 Hodges C, Bintu L, Lubkowska L, Kashlev M, Bustamante C. Nucleosomal fluctuations govern the transcription dynamics of RNA polymerase II. Science 2009; 325:626-628.

15 Kornblihtt AR, Schor IE, Allo M, Blencowe BJ. When chromatin meets splicing. Nat Struct Mol Biol 2009; 16:902903.

16 Meissner A, Mikkelsen TS, Gu H, et al. Genome-scale DNA methylation maps of pluripotent and differentiated cells. Nature 2008; 454:766-770.

17 Lorincz MC, Dickerson DR, Schmitt M, Groudine M. Intragenic DNA methylation alters chromatin structure and elongation efficiency in mammalian cells. Nat Struct Mol Biol 2004; 11:1068-1075.

18 Shukla S, Kavak E, Gregory M, et al. CTCF-promoted RNA polymerase II pausing links DNA methylation to splicing. Nature 2011; 479:74-79.

19 Luco RF, Pan Q, Tominaga K, Blencowe BJ, Pereira-Smith OM, Misteli $\mathrm{T}$. Regulation of alternative splicing by histone modifications. Science 2010; 327:996-1000. 